\title{
Tratamento cirúrgico de cisto ovariano roto em paciente com síndrome de Bernard-
}

\section{Soulier}

Surgical treatment of ruptured ovarian cyst in a patient with Bernard-Soulier syndrome

Tratamiento quirúrgico de quiste ovárico roto en paciente con síndrome de Bernard-Soulier

Recebido: 04/01/2022 | Revisado: 09/01/2022 | Aceito: 01/02/2022 | Publicado: 03/02/2022

\author{
João Victor Alcântara Pires \\ ORCID: https://orcid.org/0000-0003-0659-5749 \\ Universidade do Estado de Mato Grosso, Brasil \\ E-mail: joao.pires@unemat.br \\ Julliana Ferrari Campêlo Libório de Santana \\ ORCID: https://orcid.org/0000-0002-7773-3398 \\ Universidade do Estado de Mato Grosso, Brasil \\ E-mail: julliana.ferrari@unemat.br \\ Etienny de Brito Dias Fernandes \\ ORCID: https://orcid.org/0000-0002-3885-0014 \\ Universidade do Estado de Mato Grosso, Brasil \\ E-mail: etienny.brito@unemat.br \\ Nadia Cristina Berton \\ ORCID: https://orcid.org/0000-0002-1269-2927 \\ Universidade do Estado de Mato Grosso, Brasil \\ E-mail: nadia.berton@unemat.br \\ Isabela Furlan Franchello \\ ORCID: https://orcid.org/0000-0001-9160-2231 \\ Universidade do Estado de Mato Grosso, Brasi \\ E-mail: isabela.franchello@unemat.br \\ Rubia Martinez Santos \\ ORCID: https://orcid.org/0000-0002-2636-2013 \\ Universidade do Estado de Mato Grosso, Brasil \\ E-mail: rubia.martinez@unemat.br \\ Camila Paixão Marques \\ ORCID: https://orcid.org/0000-0002-6447-3611 \\ Hospital Regional $\operatorname{Dr}^{\circ}$ Antônio Fontes, Brasil \\ E-mail: camilapaixao2019@gmail.com \\ Letícia Cristino Francisco \\ ORCID: https://orcid.org/0000-0002-8979-0867 \\ Hospital Regional $\mathrm{Dr}^{\mathrm{o}}$ Antônio Fontes, Brasil \\ E-mail: lecristino@hotmail.com \\ Renata Fornaciari Lara \\ ORCID: https://orcid.org/0000-0002-3390-4159 \\ Hospital Regional $\operatorname{Dr}^{\circ}$ Antônio Fontes, Brasil \\ E-mail: re.flara@gmail.com
}

\begin{abstract}
Resumo
Introdução: A síndrome de Bernard-Soulier (SBS) é uma doença hematológica e hereditária rara que possui uma prevalência estimada em 1:1.000.000. Suas anormalidades são decorrentes da expressão defeituosa do complexo glicoproteico GPIb/IX/V- receptor plaquetário que é crucial para o estabelecimento da hemostasia primária. Objetivo: Relatar um caso raro de cisto ovariano roto em portador da Síndrome de Bernard-Soulier. Metodologia: estudo descritivo, transversal, que consiste na elaboração de um relato de caso, elaborado a partir de uma análise do prontuário de um paciente portador da Síndrome de Bernard-Soulier que evoluiu com cisto ovariano roto. Para embasamento teórico foram utilizados artigos indexados em português e inglês na base de dados PubMed. Foram considerados os seguintes descritores: Ovarian Cysts, Bernard-Soulier Syndrome, e Abdomen Acute. Conclusão: Considerando a frequência e a gravidade dos eventos hemorrágicos como aspectos muito variáveis entre os pacientes portadores de SBS é necessário que o cirurgião sempre tenha em mente distúrbios hematológicos subjacentes ao se deparar com um possível abdome agudo hemorrágico.
\end{abstract}

Palavras-chave: Cistos ovarianos; Síndrome de Bernard-Soulier; Abdome agudo.

\section{Abstract}

Introduction: the Bernard-Soulier Syndrome (BSS) is a rare inherited bleeding disorder witch the prevalence is estimated at 1:1,000,000. Its abnormalities are due to defective expression of the glycoproteic complex GPIb/IX/V - the platelets 
receptor that is crucial for the establishment of the prime hemostasis. Objective: report a rare case of a ruptured ovarian cyst in a patient with Bernard-Soulier Síndromes. Methodology: it's a descriptive and transversal study that consists of the formulation of a case report, built from the analysis of a patient's medical record with BSS, who evolved with ruptured ovarian cyst. For theoretical foundation we used articles indexed in PubMed's database written in portuguese and english. The following descriptors were considered: Ovarian Cysts, Bernard-Soulier Syndrome and Abdomen Acute. Conclusion: considering the frequency and severity of the hemorrhagic events as highly variable aspects among patients with BSS it is necessary that the surgeon always keep in mind the underlying hematological disorders when faced with a possible hemorrhagic acute abdomen.

Keywords: Ovarian cysts; Bernard-Soulier Syndrome; Abdomen acute.

\section{Resumen}

Introducción: el Síndrome de Bernard-Soulier (SBS) es un trastorno hemorrágico hereditario poco frecuente cuya prevalencia se estima en 1:1.000.000. Sus anomalías se deben a la expresión defectuosa del complejo glicoproteico $\mathrm{GPIb} / \mathrm{IX} / \mathrm{V}$, el receptor de plaquetas que es crucial para el estabelecimiento de la hemostasia principal. Objetivo: reportar un caso raro de ruptura de un quiste ovárico en uma paciente con Síndrome de Bernard-Soulier. Metodología: es un estudio descriptivo y transversal que consiste en la formulación de un reporte de caso, construido a partir del análisis de la historia clínica de una paciente con SBS, que evolucionó con ruptura de quiste de ovario. Para la fundamentación teórica utilizamos artículos indexados en la base de datos de PubMed escritos en português e inglés. Se consideraron los siguientes descriptores: Ovarian Cysts, Bernard-Soulier Syndrome, y Abdomen Acute.. Conclusión: considerando la frecuencia y severidad de los eventos hemorrágicos como aspectos altamente variables entre los pacientes con SBS, es necessario que el cirurjano tenga siempre presentes los trastornos hematológicos subyacentes ante un posible abdomen agudo hemorrágico.

Palabras clave: Quiestes ováricos; Síndrome de Bernard-Soulier; Abdomen agudo.

\section{Introdução}

Bernard-Soulier (SBS) consiste em um distúrbio autossômico recessivo raro com incidência de menos de 1 a cada 1 milhão de pessoas, que causa uma disfunção da adesão plaquetária por meio de um defeito no complexo Ib/IX/V da glicoproteína que se liga ao Fator de Von Willebrand (FVW) endotelial, o que impede o processo de coagulação de ocorrer normalmente. Tipicamente, ocorre trombocitopenia, plaquetas anormalmente grandes e disfuncionais, o que as impede de agregarem-se à ristocetina, mas permite a agregação normal com a ADP, colágeno e adrenalina. (Almeida et al., 2003; Almomani \& Mangla, 2021; Andrews \& Berndt, 2013; Araújo et al., 2002; Berndt \& Andrews, 2011; Bhadra \& Chacraborty, 2020; Feitosa Júnior, 2018).

As manifestações clínicas dessa síndrome incluem o sangramento mucocutâneo percebido desde a infância, apresentados como hematomas espontâneos, principalmente epistaxe, sangramento gengival e, para as mulheres, menorragia. Também é esperado que pacientes traumatizados ou em pós-operatório apresentem sangramentos de difícil controle. (Araújo et al., 2002; Berndt \& Andrews, 2011; Bhadra \& Chacraborty, 2020).

O cisto ovariano hemorrágico é a principal causa funcional de Abdome Agudo Ginecológico Hemorrágico. A ocorrência de cistos ovarianos é uma condição frequente em mulheres na idade fértil, sendo eles: os cistos foliculares, os cistos de corpo lúteo e os cistos tecaluteínicos, os cistos funcionais $24 \%$ dos CO, cistos benignos são $70 \%$, e malignos chegam a $6 \%$. Geralmente, esses achados são incidentais e regridem naturalmente, porém, a evolução anormal pode aumentar os cistos, armazenando sangue em seu interior, podendo então haver rotura e peritonite (Feitosa Júnior, 2018; Giuliano et al., 2020).

Nesse contexto, Bhadra e Chakraborty (2020), em seu relato de caso, documentou uma paciente portadora de SBS com uma menorragia incontrolável. Anteriormente, ela teve um diagnóstico de cisto ovariano aos 14 anos e o laudo histopatológico mostrou cisto endometriótico. A possível relação com o presente estudo se dá pela ocorrência do cisto ovariano em ambos os casos, justificando o presente relato. O objetivo, portanto, é relatar um caso de abdome agudo ginecológico hemorrágico cuja etiologia estava em um cisto ovariano roto, tendo como complicação a coagulopatia de Bernard-Soulier. 


\section{Metodologia}

Relato de caso detalhando-se, em específico, uma manifestação clínica referente a ocorrência de cisto ovariano roto associado à Síndrome de Bernard-Soulier, análise de seu diagnóstico e conduta baseada na literatura publicada digitalmente, para que haja mais subsídios para o tratamento de pacientes dentro desta condição. O estudo está protocolado sob o número do parecer 5.166.407, do Comitê de Ética em Pesquisa da Universidade do Estado de Mato Grosso, da cidade de Cáceres, Mato Grosso, Brasil. Para tanto, os critérios de ética da Declaração de Helsinque foram respeitados e o Termo de Consentimento Livre e Esclarecido foi assentido pela paciente em questão (Freitas-Dias, 2017).

Caso Clínico:

Paciente do sexo feminino, com 16 anos de idade, internada em unidade de referência em trauma e emergência. Referia quadro de dor e distensão abdominal difusa, associadas a astenia, mialgia, cefaleia frontal, dispneia, cianose central e periférica. Durante a entrevista médica, relatou ter o diagnóstico de Síndrome de Bernard-Soulier em acompanhamento com um hematologista. Ao exame clínico, apresentava médio estado geral, hipocorada +++/4+, PA: 90/60 mmHg, FC: 118 bpm, sinais de choque hemorrágico classe II, com abdome globoso moderadamente distendido e com sinais de irritação peritoneal. O laudo de ultrassonografia transvaginal, trazido pela paciente, relatava ascite e massa anexial esquerda a esclarecer, sugestiva de cisto ovariano hemorrágico roto. Solicitou-se a realização de exames laboratoriais que demonstraram pancitopenia, com contagem de plaquetas de $13.000 / \mathrm{mm}^{3}$, hemácias de $1,58 \mathrm{mi} / \mu \mathrm{L}$, hemoglobina com valor de $4,8 \mathrm{~g} / \mathrm{dL}$ e leucócitos de $3.970 / \mathrm{mm}^{3}$, além de um beta hCG, que teve resultado negativo. Realizou-se exame de tomografia computadorizada de abdome superior e pelve com contraste (Figura 1) em que se observou fino derrame pleural bilateral, atenuação heterogênea de ovário esquerdo sugestiva de cisto ovariano roto, bem como volumosa quantidade de líquido na cavidade abdominal. Foi submetida à estabilização hemodinâmica com monitorização contínua e hemoterapia, por meio de concentrado de hemácias fenotipadas, plasma fresco congelado e concentrado de plaquetas randômicas. A acompanhante da paciente foi informada acerca da gravidade do caso e da necessidade de abordagem cirúrgica após a transfusão especializada. Foram realizados novos exames laboratoriais após a instauração do tratamento, que constataram melhora do quadro, com valores de plaquetas de 20.000/mm², hemácias de 3,33 $\mathrm{mi} / \mu \mathrm{L}$, hemoglobina de $10 \mathrm{~g} / \mathrm{dL}$ e leucócitos de 7.120/. $\mathrm{mm}^{3}$. Optou-se, então, pela conduta cirúrgica por meio de laparotomia exploradora com incisão mediana xifo-púbica. No inventário da cavidade, observou-se a presença de cisto ovariano hemorrágico roto à esquerda, considerável quantidade de sangue na cavidade, aproximadamente 1.000 mL, e grande quantidade de coágulos. Foi realizada ressecção do cisto e rafia do ovário esquerdo, bem como lavagem repetitiva da cavidade e aspiração completa do líquido. Após a cirurgia, foram prescritos sintomáticos e solicitados exames laboratoriais diários para o acompanhamento do caso. A paciente apresentou melhora progressiva dos valores evidenciados no hemograma e evoluiu para um quadro hemodinamicamente estável. Sem mais intercorrências, recebeu alta médica no quinto dia de pós-operatório, com orientações para o seguimento ambulatorial do quadro. 
Figura 1. Tomografia computadorizada de abdome superior e pelve mostrando imagem sugestiva de cisto ovariano hemorrágico roto à esquerda ( $\mathrm{A}$ - fase pré-contraste e $\mathrm{C}$ - fase tardia) e volumosa quantidade de líquido na cavidade abdominal ( $\mathrm{B}$ - fase précontraste e D - fase tardia).

(A)

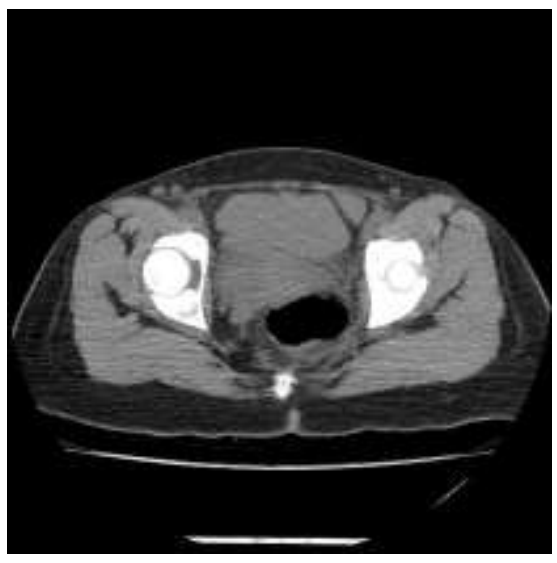

(C)

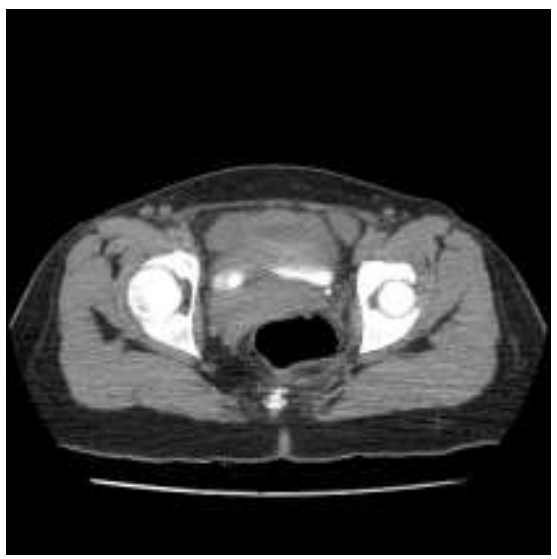

(B)

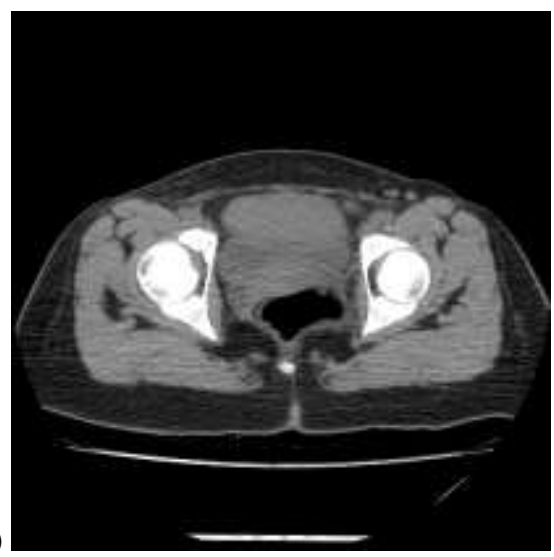

(D)

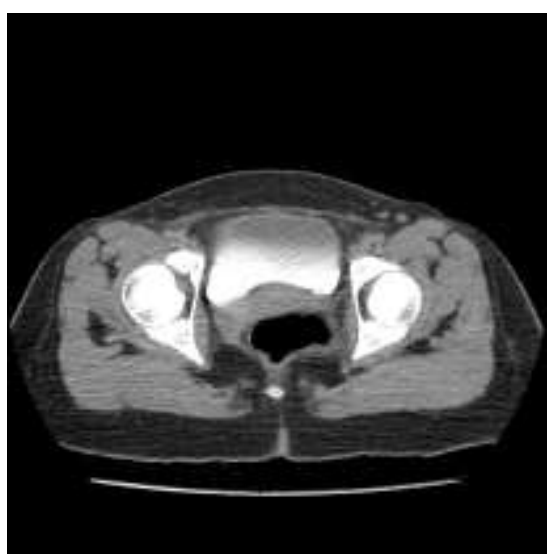

Fonte: Autores.

\section{Discussão}

A síndrome de Bernard-Soulier (SBS) é uma doença hematológica rara e hereditária, herdada, quase sempre, de forma autossômica recessiva (Almomani \& Mangla, 2021; Andrews \& Berndt, 2013; Araújo et al., 2002; Berndt \& Andrews, 2011; Boecklemann et al., 2017; Grainger et al., 2018; Kunishima et al., 2002; Ozelo et al., 2005; Savoia et al., 2014), sendo mais comum em famílias onde há relações consanguíneas (Greinacher et al., 1993; Gupta et al., 2020; Kunishima et al., 2002; Lanza, 2006; Savoia et al., 2014) com uma prevalência estimada de 1:1.000.000 (Almomani \& Mangla, 2021; Boeckelmann et al., 2017; Grainger et al., 2018; Kunishima et al., 2002; Ozelo, Svirin \& Larina, 2005; Savoia et al., 2014; Afshar-Kaharghan \& López, 1997), podendo esse número ser subestimado devido à formulação de diagnósticos incorretos e à subnotificação. Clinicamente a síndrome manifesta-se por tempo de sangramento aumentado, plaquetas gigantes e plaquetopenia (Almeida et al., 2003; Almomani \& Mangla, 2021; Andrews \& Berndt, 2013; Araújo et al., 2002; Berndt \& Andrews, 2011; Bhadra \& Chacraborty, 2020; Boecklemann et al., 2017; Farhan \& Igbal, 2019; Grainger et al., 2018; Greinacher et al., 1993; Gupta et al., 2020; Kunishima et al., 2002; Lanza, 2006; Ozelo et al., 2005; Reisi, 2020; Savoia et al., 2014), sendo identificada desde o nascimento ou na primeira infância, em função do aparecimento de púrpura refratária aos tratamentos convencionais, presença de hematomas pelo corpo, sangramento gengival, hemorragias excessivas em procedimentos cirúrgicos e, mais tardiamente, menorragia nas pacientes do sexo feminino (Feitosa Júnior, 2018; Boeckelmann et al., 2017; Greinacher et al., 1993; Gupta et al., 2020).

Em um pequeno número de pacientes, a SBS é herdada de forma autossômica dominante, sendo referida como SBS monoalélica (Grainger et al., 2018). Geralmente, nesses casos, a síndrome é identificada quando o paciente é adulto, uma vez 
que os sinais e sintomas costumam ser mais brandos, com redução dos episódios hemorrágicos, o que dificulta sua identificação. A variante de Bolzano (Almomani \& Mangla, 2021; Andrews \& Berndt, 2013; Grainger et al., 2018; Kunishima et al., 2002) é a mais famosa expressão heterozigótica da SBS, em que os pacientes apresentam macrotrombócitos, com ampla conservação da massa de plaquetas, apresentando, portanto, plaquetopenia leve ou inexistente (Grainger et al., 2018; Kunishima et al., 2002).

Bioquimicamente, a expressão do complexo glicoproteico GPIb/IX/V, localizado na superfície de membrana das plaquetas, é defeituosa (Almeida et al., 2003; Almomani \& Mangla, 2021; Andrews \& Berndt, 2013; Araújo et al., 2002; Berndt \& Andrews, 2011; Bhadra \& Chacraborty, 2020; Boecklemann et al., 2017; Farhan \& Igbal, 2019; Grainger et al., 2018; Greinacher et al., 1993; Gupta et al., 2020; Kunishima et al., 2002; Lanza, 2006; Ozelo et al., 2005; Reisi, 2020; Savoia et al., 2014; Afrasiabi, A. et al., 2007). O complexo GPIb/IX/V, cuja principal função é servir como receptor plaquetário para o fator de von Willebrand (vWF), é de extrema importância para o estabelecimento da hemostasia primária, mediando a adesão plaquetária aos locais de exposição subendotelial onde há lesões vasculares o que, consequentemente, resulta em coagulopatia (Grainger, Thachil \& Will, 2018). Além disso, estima-se que esse mesmo complexo possa desempenhar um papel importante na síntese normal das plaquetas na medula óssea. O que fica evidente quando se observam as alterações numéricas e morfológicas características nos trombócitos dos indivíduos portadores da síndrome (Grainger et al., 2018).

O complexo GPIb/IX/V é composto de quatro glicoproteínas transmembrana, GPIb $\alpha$, GPIb $\beta$, GPIX e GPV, localizados, respectivamente, nos cromossomos 17p12, 22q11.2, 3q21 e 3q29 (Kunishima et al., 2002; Lanza, 2006). As três primeiras (GPIb $\alpha$, GPIbß e GPIX) estão diretamente associadas e são todas indispensáveis para a biossíntese do receptor, de modo que, a ausência de uma dessas subunidades reduz dramaticamente a expressão de todo o complexo. Em contrapartida, a ausência do componente GPV não afeta a expressão e/ou o funcionamento do complexo (Andrews \& Berndt, 2013; Kunishima et al., 2002; Lanza, 2006). Até o momento, quase 112 mutações foram identificadas em portadores da SBS, em que as mutações nos genes GPIb $\alpha$, GPIb $\beta$ e GPIX, representam, na devida ordem, 28\%, 28\% e 44\% dos casos (Savoia et al., 2014). Essas mutações são heterogêneas e podem ser sem sentido, deslocamento de quadrado, exclusão ou inserção (Almomani \& Mangla, 2021).

A frequência e a gravidade dos eventos hemorrágicos variam muito entre os pacientes com SBS (Boeckelmann et al., 2017; Lanza, 2006). A epistaxe é a principal manifestação clínica (Araújo et al., 2002; Ozelo et al., 2005), estando presentes também púrpuras, sangramento gengival, sangramento cutâneo, hemorragia pós-trauma, sangramentos prolongados após extrações dentárias e menorragia. Mais raramente, evidenciam-se sangramentos gastrintestinais e hematúria (Almomani \& Mangla, 2021; Boecklemann et al., 2017; Grainger et al., 2018; Kunishima et al., 2002; Lanza, 2006). Na maioria dos casos a tendência de sangramento é exacerbada em relação aos níveis de plaquetopenia, sendo a morte por SBS um desfecho muito raro (Almomani \& Mangla, 2021). Para firmar o diagnóstico sindrômico é necessária uma conjuntura de fatores: aumento do tempo de sangramento (TS), presença de macrotrombocitopenia, aglutinação induzida por ristocetina defeituosa - não corrigida por adição de plasma - e exiguidade ou ausência da glicoproteína GPIb/IX/V, identificada por citometria de fluxo, que utiliza um painel de anticorpos monoclonais específicos (CD42 a-d) (Gupta et al., 2020; Lanza, 2006). Os testes laboratoriais devem incluir hemograma e esfregaços sanguíneos. A contagem de plaquetas, em geral, varia de 20.000 a 100.000/mm³ (Lanza, 2006).

A Púrpura Trombocitopênica Imune (PTI) é o diagnóstico diferencial mais importante, de modo que muitos pacientes recebem tal diagnóstico e são submetidos a tratamentos com imunoglobulina intravenosa, glicocorticóides ou até mesmo esplenectomia, sem que haja resposta, antes que se estabeleça o diagnóstico de SBS (Grainger et al., 2018; Kunishima et al., 2002; Lanza, 2006). Outros diagnósticos diferenciais importantes são a Doença de von Willebrand (DvW) identificada pela resposta à aglutinação induzida por ristocetina, o que não ocorre na SBS; Anormalidade de May-Hegglin; Doenças da Cadeia Pesada 9 da Miosina (MYH-9); Síndrome das Plaquetas Cinzentas; Desordem Plaquetária de Paris-Trousseau (PTPD); Síndrome de DiGeorge (DGS) e Macrotrombocitopenia Mediterrânea (Almomani \& Mangla, 2021). 
O manejo do paciente com SBS começa pela educação em saúde, através de fornecimento de orientações aos pacientes e seus familiares direcionada à boa higiene bucal, a não praticar esportes com alto risco traumático e em como usar a pressão aplicada nos casos de epistaxe. Além disso, esses pacientes devem ser alertados quanto ao uso de medicamentos como antinflamatórios não esteroidais, principalmente, o ácido acetilsalicílico (aspirina), alguns antibióticos, antihistamínicos e drogas psicoativas. Como medida de suporte, pode-se fazer reposição de ferro para a anemia secundária aos episódios hemorrágicos (Grainger et al., 2018).

Como medidas específicas de abordagem na SBS, a terapia antifibrinolítica, com ácido tranexâmico, é a mais largamente utilizada. Tendo se provado segura e efetiva para controle de sangramentos cutaneomucosos, sangramentos pós-procedimentos dentários, epistaxe e menorragia (Grainger et al., 2018). Uma abordagem terapêutica alternativa é a utilização da desmopressina (DDAVP), um medicamento análogo à vasopressina, que em alguns relatos de caso (Greinacher et al., 1993) demostrou reduzir o tempo de sangramento em pacientes com SBS. Contudo, devido aos seus efeitos colaterais, a utilização dessa droga tem sido desencorajada.

Para pacientes que serão submetidos a grandes procedimentos cirúrgicos ou que apresentem hemorragias graves ou ainda naqueles que se mantiveram refratários à terapia antifibrinolítica, a transfusão de concentrado de plaquetas é o tratamento de primeira linha (Almeida et al., 2003; Almomani \& Mangla, 2021; Grainger et al., 2018; Gupta et al., 2020; Kunishima et al., 2002; Lanza, 2006; Ozelo, Svirin \& Larina, 2005). Entretanto, atenção especial deve ser dada a esse procedimento. Devido ao risco de desenvolvimento de aloanticorpos contra o complexo glicoproteico da superfície dos trombócitos, deve-se transfundir plaquetas com antígenos leucocitários humanos (HLA) compatíveis aos do indivíduo receptor, no intuito de se minimizar tal risco (Almomani \& Mangla, 2021; Boeckelmann et al., 2017; Grainger et al., 2018; Gupta et al., 2020). Nos pacientes que já desenvolveram os aloanticorpos, e que necessitam de transfusão, grandes doses de concentrado de plaquetas devem ser coadministradas com fator VIIa recombinante (Grainger et al., 2018). Apesar o fator VIIa recombinante ainda não ser aprovado para o tratamento do SBS, existem relatos exitosos (Almeida et al., 2003; Ozelo, Svirin \& Larina, 2005) de sua utilização no manejo de episódios hemorrágicos graves ou recorrentes, além de fornecer cobertura hemostática a pacientes durante a realização de procedimentos cirúrgicos, tornando-o uma opção viável, principalmente, para pacientes que desenvolveram anticorpos ou que se tornaram não responsivos à transfusão de plaquetas (Ozelo et al., 2005).

O prognóstico dos pacientes portadores da Síndrome de Bernard-Soulier geralmente é bom (Almomani \& Mangla, 2021), principalmente se receberem os cuidados adequados e em tempo hábil e se seguirem as orientações fornecidas durante o processo de educação em saúde, como manter uma boa higiene bucal e evitar atividades que possam gerar lesões (Grainger, Thachil \& Will, 2018) traumáticas. Além disso, recomenda-se que o paciente tenha uma pulseira de identificação (Almomani \& Mangla, 2021; Grainger et al., 2018), que sirva como alerta à equipe de saúde, em serviços de emergência, quanto à natureza clínica da síndrome caso o paciente venha a sofrer algum trauma.

\section{Conclusão}

Nos portadores de Síndrome de Bernard-Soulier, a frequência e a magnitude dos episódios hemorrágicos são muito variáveis. A hemorragia pode ser proveniente de cisto parovariano roto durante a menacme, entretanto a literatura médica atual ainda não postula que existe uma correlação direta entre a Síndrome e o quadro clínico, motivo pelo qual se pauta a importância da realização de novas pesquisas com esse enfoque clínico-patológico.

Todavia, mesmo com a necessidade de mais estudos sobre o tema, ao deparar-se com paciente apresentando exuberante hipótese diagnóstica de abdome agudo hemorrágico, é imprescindível que o profissional sempre esteja ciente de quaisquer distúrbios hemorrágicos hematológicos subjacentes que o paciente apresente previamente. Para que assim, o diagnóstico seja otimizado e possíveis complicações sejam evitadas, tendo em vista a gravidade do quadro. 


\section{Referências}

Afrasiabi, A. et al. (2007). Genetic characterization of patients with Bernard-Soulier syndrome and their relatives from Southern Iran. Platelets. 18(6), 409-13.

Afshar-Kaharghan, V., \& López, J. A. (1997) Bernard-Soulier Syndrome Caused by a Dinucleotide Deletion and Reading Frameshift in the Region Encoding the Glycoprotein Ib $\alpha$ Transmembrane Domain. Blood. 90(7), 2634-43.

Almeida, A. M., Khair, K., Hann, I., \& Liesner, R. (2003). The use of recombinant factor VIIa in children with inherited platelet function disorders. British Journal Of Haematology. 121(3), 477-81. http://dx.doi.org/10.1046/j.1365-2141.2003.04286.x.

Almomani, M. H., \& Mangla, A. (2021) Bernard Soulier Syndrome. Statpearls. 1(1), 1-8.

Andrews, R., \& Berndt, M. (2013). Bernard-Soulier Syndrome: an update. Seminars In Thrombosis And Hemostasis. 39(6), 656-62. http://dx.doi.org/10.1055/s0033-1353390.

Araújo, S., Alves, W., Bueno, L. M., Dionísio, C., \& Siqueira, P. H. (2002). Epistaxe como manifestação frequente da síndrome de Bernard-Soulier. Revista Brasileira de Otorrinolaringologia. 68(2), 284-86. http://dx.doi.org/10.1590/s0034-72992002000200021.

Berndt, M. C., \& Andrews, R. K. (2011). Bernard-Soulier syndrome. Haematologica. 96(3), 355-59. http://dx.doi.org/10.3324/haematol.2010.039883.

Bhadra, D., \& Chakraborty S. (2020). Bernard-Soulier syndrome (BSS) with uncontrollable menorrhagia. Asian J Transfus Sci. 14(1), 93-5. https://www.ajts.org/article.asp?issn=0973-6247; year=2020; volume=14;issue=1; spage=93; epage=95; aulast=Bhadra.

Boeckelmann, D, Hengartner, H., Greinacher, A., Nowak-Göttl, U., Sachs, U., Peter, K., Sandrock-lang, K., \& Zieger, B. (2017). Patients with Bernard-Soulier syndrome and different severity of the bleeding phenotype. Blood Cells, Molecules, And Diseases. 67(1), 69-74. http://dx.doi.org/10.1016/j.bcmd.2017.01.010.

Farhan, S., \& Iqbal I, A. N. (2019). Bernard Soulier Syndrome: 10 years' experience at a tertiary care hospital. Pak J Med Sci. 35(3), 705-08. 10.12669/pjms.35.3.980. PMID: 31258580; PMCID: PMC6572988. https://www.ncbi.nlm.nih.gov/pmc/articles/PMC6572988/.

Feitosa Júnior, V. N. (2018). Abordagem do abdome agudo ginecológico: trabalho de conclusão de residência médica (residência médica em cirurgia geral). Hospital Geral de Fortaleza, http://extranet.hgf.ce.gov.br/jspui/bitstream/123456789/358/1/2018_TCR_CirurgiaGeral_FeitosaJ\%c3\%banior_VAL.pdf.

Freitas-Dias, R. (2017). Metodologia Científica: um guia prático para profissionais da saúde. Editor: Ricardo Freitas

Giuliano, L.C., Luciano, L.S., Portilho, S. S., Waldrich, L., Arruda, J. F., \& São Tiago, L. E. (2020). Diagnóstico ocasional de cisto ovariano durante procedimento de oclusão percutânea de comunicação interatrial. J Transcat Intervent. https://doi.org/10.31160/JOTCI202028A20200023.

Graigner, J. D., Thachil, J., \& Will, A. M. (2018). How we treat the platelet glycoprotein defects; Glanzmann thrombasthenia and Bernard Soulier syndrome in children and adults. British Journal Of Haematology. 182(5), 621-32. http://dx.doi.org/10.1111/bjh.15409.

Greinacher, A. et al. (1993). Evidence that DDAVP transiently improves hemostasis in Bernard-Soulier syndrome independent of von Willebrand-Factor. Annals Of Hematology. 67(1), 149-51.

Gupta, A. et al. (2020). A case report of Bernard Soulier Syndrome and its management in minor oral surgical procedures. International Journal Of Current. 9(12), 13545-48.

Kunishima, S., Kamiya, T., \& Saito, H. (2002). Genetic Abnormalities of Bernard-Soulier Syndrome. International Journal Of Hematology. 76(4), 319-27. http://dx.doi.org/10.1007/bf02982690.

Lanza, F. (2006). Bernard-Soulier syndrome (Hemorrhagiparous thrombocytic dystrophy). Orphanet Journal Of Rare Diseases. 1(1), 1-6. http://dx.doi.org/10.1186/1750-1172-1-46.

Ozelo, M. C., Svirin, P., \& Larina, L. (2005). Use of recombinant factor VIIa in the management of severe bleeding episodes in patients with Bernard-Soulier syndrome. Annals Of Hematology. 84(12), 816-22. http://dx.doi.org/10.1007/s00277-005-1080-y.

Reisi, N. (2020). Bernard-Soulier syndrome or idiopathic thrombocytopenic purpura: A case series. Caspian J Intern Med. 11(1), 105-09. 10.22088/cjim.11.1.105. https://www.ncbi.nlm.nih.gov/pmc/articles/PMC6992729/.

Savoia, A., Kunishima, S., Rocco, D. de., Zieger, B., Rand, M. L., Pujol-Moix, N., Caliskan, U., Tokgoz, H., Pecci, A., \& Noris, P. (2014). Spectrum of the Mutations in Bernard-Soulier Syndrome. Human Mutation. 35(9), 1033-45. http://dx.doi.org/10.1002/humu.22607. 